Archives

$42 \mid 2008$

Circulations et frontières

\title{
Frontières nationales et frontières des réseaux dans l'« Espace Schengen »
}

Les migrants des pays pauvres et l'affaiblissement des modèles intégrateurs des États-nations européens

\section{Alain Tarrius}

\section{(2) OpenEdition}

1 Journals

\section{Édition électronique}

URL : http://journals.openedition.org/ccrh/3484

DOI : $10.4000 /$ ccrh.3484

ISSN : $1760-7906$

Éditeur

Centre de recherches historiques - EHESS

\section{Édition imprimée}

Date de publication : 25 avril 2008

Pagination : 283-296

ISSN : 0990-9141

\section{Référence électronique}

Alain Tarrius, «Frontières nationales et frontières des réseaux dans I'« Espace Schengen » », Les Cahiers du Centre de Recherches Historiques [En ligne], 42 | 2008, mis en ligne le 08 novembre 2011, consulté le 19 avril 2019. URL : http://journals.openedition.org/ccrh/3484 ; DOI : 10.4000/ccrh.3484

Ce document a été généré automatiquement le 19 avril 2019.

Article L.111-1 du Code de la propriété intellectuelle. 


\section{Frontières nationales et frontières des réseaux dans l'" Espace Schengen "}

Les migrants des pays pauvres et l'affaiblissement des modèles intégrateurs des États-nations européens

\section{Alain Tarrius}

De nouvelles formes migratoires transnationales, fédérant en réseaux les initiatives économiques de collectifs issus de pays pauvres, apparaissent depuis une vingtaine d'années en Europe. J'ai eu l'opportunité d'identifier dès 1984 de telles innovations économiques et sociales dans le Bassin méditerranéen occidental, puis de suivre leur développement. C'est ainsi que mes recherches, me conduisirent à tenter de renouveler l'analyse des migrations en montrant comment se construisent aujourd'hui de nouveaux «territoires circulatoires» transnationaux qui font la trame - «par le bas»- de la mondialisation. Ces recherches m'ont forcé à reconsidérer à la fois les formes de cette mondialisation, en mettant en évidence l'émergence d'un nouveau «capitalisme nomade ", à l'initiative de migrants entrepreneurs, et les cadres de pensée au moyen desquels nous appréhendons les migrations : la dialectique de l'individu migrant et de l'État, dont la résolution selon le schème de l'intégration semble concurrencée aujourd'hui par d'autres formes d'appartenance et d'attachement des collectifs à leurs territoires est au cœur de ces investigations.

\section{De Marseille au Maroc, les « contrebandiers de la mondialisation »}

\section{L'exemplarité marseillaise}

2 Le cas de Marseille et des réseaux des économies souterraines entre le Maghreb et le Bassin méditerranéen occidental est particulièrement révélateur de la genèse de ces formes, de leur incessante transformation. Il expose un modèle déjà mondialisé 
3 Dans les années 1985-1987, 350 commerces tenus par des migrants d'origine maghrébine, essentiellement algérienne, œuvraient dans le quartier historique central, en déshérence, de Belsunce. On dénombrait alors 81 familles propriétaires des fonds de commerces, dont 39 algériennes, 27 tunisiennes et 15 marocaines. 700 000personnes, dont environ 300000 immigrants en Europe, transitaient annuellement par ce quartier et y effectuaient toute sorte d'achats qui compensaient les difficultés d'approvisionnement des nations maghrébines. Le chiffre d'affaires de ces commerces évalué par la SEDES (Caisse des Dépôts et Consignations) en 1987 était d'environ trois milliards de francs, compte non tenu des contre-façons (pièces détachées, vêtements,...) ni des voitures passées en contrebande. Quatre événements contribuèrent, à la fin des années quatre-vingt, et au début des années quatre-vingt-dix, à une transformation de ce dispositif commercial :

4 - la limitation des visas entre l'Algérie et la France, lors du premier passage de Charles Pasqua au Ministère de l'Intérieur ;

5 - les effets de la crise politique algérienne : le Front Islamique du Salut (FIS) tentant de prélever un impôt « révolutionnaire » sur les commerçants algériens, ceux-ci passèrent en grand nombre la gérance de leurs commerces à des Marocains ;

6 - la grande expansion migratoire marocaine, qui transforma l'histoire sociale de l'Espagne et de l'Italie, de nations d'émigration en nations d'immigration, et, toujours forte, déstabilise particulièrement les politiques et pratiques législatives de ces nations vis-à-vis des étrangers. Les centralités marocaines des réseaux de migrants commerciaux se sont rapidement généralisées de Bruxelles, place traditionnelle, et de l'axe Maroc, Madrid, Irun, Bordeaux, Paris, vers Marseille, Milan, Naples, Francfort, et diverses villes espagnoles, c'est-à-dire vers un nouvel axe de circulations circum-méditerranéen; ce mouvement se déclencha à la fin des années quatre-vingt à partir, d'abord, de collaborations à Bruxelles entre Marocains et Turcs ;

7 - la densification des réseaux d'économies souterraines de l'Est européen et leur connexion avec les réseaux méditerranéens orientaux et occidentaux ou maghrébins ;

$8 \mathrm{Au}$ fur et à mesure de ces événements les logiques de fonctionnement en réseaux l'emportèrent sur celles de place marchande unique avec ses logistiques de transports de lieu à lieu. Les Algériens des commerces internationaux, ayant passé la main aux Marocains et aux Tunisiens, se replièrent plutôt sur des commerces de proximité, dans les marchés publics ou dans les quartiers des villes relativement mal desservis, cependant que leurs successeurs accentuaient forte-ment la nature et la forme de ce dispositif commercial vers l'internationalité. Les entrepreneurs maghrébins de Marseille, au lieu de gérer localement en moyenne quatre commerces locaux, ouvrirent des entrepôts de chargement de marchandises ou encore des magasins en plus grand nombre le long des espaces supports aux réseaux. Ils acquirent ainsi une plus grande efficience commerciale, mobilisant désormais des «fourmis» domiciliées tout au long des parcours, qui transportèrent désormais, dans des fourgons et des voitures, des marchandises au cours d'incessants va et vient. À Marseille la visibilité des commerçants installés à Belsunce devint évidemment moindre, alors même que leur influence et leur richesse s'accroissaient. En fait, soixante-seize familles des quatre-vingt-une présentes en 1985, opèrent toujours dans le centre de Marseille (17 d'entre elles, algériennes, ont confié des gérances à des Marocains) et ont été rejointes par 43 familles marocaines et 2 tunisiennes. Le dispositif marseillais compte donc actuellement (enquêtes automne 2.000) 126 familles de commerçants (22 algériennes, 29 tunisiennes et 75 marocaines) qui possèdent en 
moyenne sept commerces ou entrepôts de chargement le long des réseaux, de l'Italie au Maroc, soit plus de 800 établissements. Ce qui est perçu de ces changements, à partir de critères «localistes» de description et d'évaluation, est désigné comme régression du dispositif commercial marseillais, alors que son influence est plus forte que jamais, enrichie par l'émergence, qu'elle provoque et gère en grande partie, de nouvelles centralités dans les pays voisins. La piste des Algériens, dominants à Belsunce jusqu'en 1989, mène aujourd'hui aux marchés publics locaux ou à la rationalisation des trabendes par containers pour la seule Algérie. Celle des autres migrants commerçants permet d'identifier le monde comme origine ou destination des échanges. Le déploiement des petits migrants, "fourmis » de ces commerces internationaux, est tel qu'entre 1991 et 1995 les véhicules mobilisés pour les transports de marchandises passèrent de 1.700 pour un aller-retour mensuel à 42.000 pour deux allers-retours mensuels, permettant à environ 192.000 personnes domiciliées dans le Sud de la France, de tirer des revenus notables de cette grande proximité avec leur pays d'origine, le Maroc. Marseille, Perpignan, Alicante et d'autres étapes encore, prospèrent en même temps. Pour ce qui est précisément d'Alicante, nos enquêtes depuis janvier $1999^{1}$ nous ont permis d'identifier, parmi les quarante-cinq commerces ou entrepôts ouverts depuis 1997 à Crévillent, à environ trente kilomètres du port levantin, dix-sept points de vente et de chargement gérés comme succursales par des entrepreneurs toujours installés à Marseille.

Ces populations de migrants, qui développent leurs sociabilités autour des réseaux commerciaux internationaux, n'affrontent jamais frontalement les sociétés locales, dans l'appropriation d'espaces urbains: la tension toujours pré-sente entre appartenances lointaines et proches leur confère une grande fluidité, labilité, dans le choix de leurs étapes. Les commerçants savent toujours se re-localiser, suivis par les cohortes de « fourmis » circulantes, dans les espaces des réseaux ou de la ville.

\section{De Marseille au Maroc : territoires et sociabilités autres}

10 Nous avons pu identifier, entre Marseille et le Maroc, quelques tracés originaux de frontières territoriales et des rapports sociaux spécifiques aux réseaux de migrants commerçants. Nos enquêtes se sont en premier lieu déroulées au cours d'accompagnements de circulants, et donc d'observations directes, et d'entretiens avec plusieurs «notaires informels $»^{2}$ le long des itinéraires circum-méditerranéens. La frontière entre la France et l'Espagne ne fait guère sens pour ces populations : trois types de passages existent, l'autoroute, la nationale et les chemins carrossables. Sur l'autoroute la frontière du Perthus n'est plus la traditionnelle barrière gardée par des policiers et des douaniers postés; elle s'est transformée en une vaste pénétrante franco-espagnole, de Gérone à Montpellier, avec des brigades "volantes " de douaniers susceptibles de se manifester dans n'importe quelle sortie tout au long de ces trois-cents kilomètres. Les postes de douane sur les nationales, à Cerbère et au Perthus, sont désertés depuis plusieurs années et hébergent des patrouilles aléatoirement, pour quelques heures; quant aux chemins carrossables, à Banyuls et dans d'autres villages des Albères, ils permettent des passages libres, mais peu confortables sur pistes forestières. Les changements d'itinéraire en passage de frontière n'interviennent que dans les phases dites de "vigipirate renforcé", afin d'éviter les longues files de véhicules lorsque la « fonction-barrière » de la frontière reprend le dessus au poste autoroutier du Perthus. Dans ces cas, ce sont généralement des militaires qui fouillent les véhicules à la recherche d'armes ou d'autres marchandises dangereuses. Les migrants-commerçants sont peu concernés par ces dispositions, sinon par les pertes de temps occasionnées par ces arrêts et les fouilles qui en 
résultent. Cette évolution de la frontière est de peu d'importance pour les migrantscommerçants: d'une part, ils circulent dans le sens des sorties du territoire et donc leurs chargements sont moins observés et sollicités que dans le sens entrant et d'autre part, ils savent combiner de façon adéquate les trois types de voies de passage.

11 En revanche, nous avons pu identifier une frontière "morale » et territoriale forte à la hauteur d'Alicante et de Murcia. Elle a pour fonction de contenir dans l'Andalousie toutes sortes de criminalités liées à la migration en Europe par le Maroc. Alicante et, dans sa périphérie, Crévillent, petit village sur l'autoroute hébergeant un dispositif commercial lié à celui de Marseille, sont les étapes ultimes des itinéraires vers le Maroc: les "fourmis» du commerce trans-national s'y arrêtent pour les derniers achats, en particulier de tapis et de nourriture, avant la traversée rapide, sans arrêts, de l'Andalousie et l'embarquement à Algésiras ou à Malaga. Alicante fait frontière maritime également avec l'Algérie (Oran) et joue, elle aussi, un rôle de forte complémentarité avec Marseille. La contention entre Murcia, Crévillent et Alicante, du " chaos Andalou », selon les termes d'un notaire informel résident à Murcia, est le fait non seulement des commerçants installés à disposition des circulants, mais encore, et surtout, de membres de mouvements islamistes algériens, insérés dans le dispositif commercial, qui craignent la mise en danger de leurs propres réseaux ${ }^{3}$ par des initiatives illégales de Marocains installés dans ces confins troubles, criminogènes, que représente désormais l'Andalousie.

\section{Lieu, mouvement et hiérarchies identitaires}

\section{Temporalités organisatrices des territoires}

12 L'apparition de collectifs, plus ou moins stables et durables, où les critères d'identification des individus, la hiérarchie des préséances, sont tributaires des temporalités, des fluidités, des mobilités, et plus précisément des capacités d'appartenances multiples de chacun, provoque probablement en premier lieu la modification de vieux schèmes comportementaux. L'ordre que l'on a si longtemps présenté comme universellement édificateur des légitimités identitaires, «l'attachement au lieu», et les diverses hiérarchies qu'il génère, ne fait plus réellement sens dans ces milieux. Ainsi sont bouleversés les rapports entre identités et altérités selon des clivages transversaux aux diverses stratifications sociales et économiques, associant sou-vent, malgré elles, des populations aux statuts et aux intérêts économiques et politiques divergents ${ }^{4}$. La notion de territoire est à redéfinir si l'on désire comprendre que des individus, des collectifs, modifient, par séquence ou dans la totalité de leur existence, les bases usuelles de l'affirmation identitaire sédentaire.

Cette notion de territoire est aussi floue que celle d'identité ; elle exige, à chaque usage, un rappel de sa définition. A minima, nous dirons ${ }^{5}$ que le territoire est une construction consubstantielle de la venue à forme puis à visibilité sociale d'un groupe, d'une communauté ou de tout autre collectif dont les membres peuvent employer un "nous " identifiant. Il est condition et expression du lien social. Le territoire est mémoire : il est le marquage spatial de la conscience historique d'être ensemble. Les États-nations ont proposé une organisation des territoires basée sur la présence de hiérarchies politiques dans la totalité de l'espace support aux sociabilités. Les réseaux que nous observons dérogent à cette construction politique et s'instituent ainsi en contre-modèles: les 
temporalités sociales y jouent un rôle premier d'organisatrices des contours territoriaux et de leur fluidité.

Pour nous donc, la mémoire en partage, qui permet d'affirmer une identité circulatoire, est extensive autant que le sont les territoires des circulations: elle n'est pas puits mnésique, elle signale non pas l'épaisseur des lieux connus, et les légitimités «notabilaires» locales, mais les moments des négociations qui permettent de porter plus loin ses initiatives, de rencontrer, de traverser plus de différences. Cette mémoire collective est avant tout souvenir des accords de parole, des échanges d'honneur, qui fluidifient les circulations, qui permettent d'échapper aux régulations étatiques formelles, de contourner les règles de construction des frontières entre territoires et entre univers de normes, celles qui disent les conditions du passage d'une sédentarité à une autre. La référence à cette mémoire collective autorise chacun à aller plus avant, à se présenter encore et encore, à s'agréger à d'autres, ou bien à les expulser de l'espace des multiples étapes et réseaux supports à l'initiative circulatoire. À Marseille en 1985, à Montpellier, Perpignan ou Barcelone en 1992, à Alicante, Crévillent, Grenade, Almeria en 2000, nous avons toujours rencontré ces réunions, dans un café ou une arrière boutique, où un «notaire informel» facilite les transactions commerciales, puis en contrôle le déroulement. Ces réunions réalisent des lieux-moments exceptionnels dans l'organisation sociale et l'affirmation identitaire de ces collectifs : c'est alors que certains, au bout de mois ou d'années d'errance, sont cooptés par les réseaux, et désormais s'ouvre à eux un univers inépuisable d'opportunités économiques, de trajectoires de réussite personnelle et familiale. C'est l'instant où fléchissent, s'effacent parfois, les barrières des différences ethniques, et le Polonais, le Bulgare, l'Italien, le Turc, le Maghrébin, l'Africain subsaharien, etc., engagent des échanges durables, partagent « une éthique intermédiaire de l'honneur ». Dès lors, la parole donnée ne peut être rendue ou reprise, qu'après une dénonciation vigoureuse par le «notaire informel » : la dérogation aux codes d'honneur toujours rappelés lors de l'entrée dans ces univers des réseaux est immédiatement sanctionnée par un redoutable exil, une exclusion radicale et rapide. Ce moment est celui de la venue à communauté, d'esprit certes, mais encore de voisinage, dans ces territoires des circulations, connectés aux sociétés locales en quelques étapes résidentielles.

\section{Des territoires de la mobilité}

Ces territoires, nous les nommons territoires circulatoires. Cette notion constate la socialisation d'espaces supports à des pratiques de mobilité. Elle introduit une double rupture dans les acceptions communes du territoire et de la circulation; en premier lieu elle nous suggère que l'ordre né des sédentarités n'est pas essentiel à la manifestation du territoire, ensuite elle exige une rupture avec les conceptions logistiques des circulations, des flux, pour investir de sens social le mouvement spatial. Le déplacement, qui ne peut dans cette perspective être considéré comme l'état inférieur de la sédentarité, confère à ceux qui en font leur principal lieu d'expression du lien social le pouvoir du nomade sur le sédentaire : la connaissance des "savoir-faire chemin », condition de la concentrationdiffusion des richesses matérielles et immatérielles, donne force sur l'ordre des sédentarités, et plus particulièrement sur sa manifestation première, l'espace urbain.

L'expansion de ces territoires, inséparable des solidarités qui les constituent en topiques d'échanges de haute densité et diversité, génère sans cesse de nouvelles connivences avec de nouveaux autres, fédérés au collectif circulatoire pour mieux transiter, atteindre des 
marchés, des emplois, des sites, de plus en plus lointains. Les différences attachées à l'ethnicité, en sont de plus en plus bannies, dès lors que se manifeste cette éthique sociale intermédiaire; en somme, l'identité commune à tous les arpenteurs des territoires circulatoires est faite de la plus grande interaction possible entre altérités... ainsi naissent les nouveaux mondes cosmopolites nomades.

La notion de "territoire circulatoire » habilite une démarche anthropologique étendue à la définition d'espaces relativement autonomes supportant des segmentations sociales, économiques et spatiales originales. La mobilité spatiale exprime donc plus qu'un mode commun d'usage des espaces : des hiérarchies sociales, des reconnaissances, qui donnent force et pouvoir, qui dissimulent aussi, aux yeux des sociétés de sédentaires, des violences et des exploitations non moins radicales. Les personnes en situation d'errance, quelles que soient leur origine et leur fortune, paient un tribut élevé pour acquérir un peu de protection des circulants maîtres de leur mobilité, des nouveaux nomades : passages de frontières à risques, clandestinités diverses, tâches pénibles sans limites autres que l'épuisement de certaines formes de travail « au noir »...

Les «petits» migrants qui rejoignent par dizaines de milliers les circulations internationales, après avoir parfois longuement tenté des trajectoires d'intégration dans les sociétés d'accueil, après aussi des errances non moins difficiles, sans papiers, sans famille, deviennent des nomades: le projet diasporique d'assimilation ne les concerne pas, celui de l'errance ne les concerne plus, ils se tiennent à distance des valeurs des sociétés d'accueil, se déploient, souvent familialement, dans des espaces internationaux qui excèdent des conditions usuelles et nécessaires à l'entrée dans les hiérarchies locales de l'identité. À l'adolescence tel jeune quittera sa famille parentale pour rejoindre, lorsque l'opportunité d'une activité se présente, tel oncle ou autre parent dans un pays européen différent.

\section{L'intégration autre}

19 Le second événement source de transformation des identités de ces collectifs, concerne l'apparition concomitante d'individus, isolés ou regroupés, souvent étrangers aux nations qui les hébergent, qui bricolent, précisément à partir de leurs expériences circulatoires, des identités métisses entre univers proches et lointains, transnationaux souvent, imposant à la classique oppositionentre les nôtres et les leurs, entre être d'ici ou de làbas, une autre forme, triadique, c'est à dire hautement processuelle : l'être d'ici, l'être de là-bas, l'être d'ici et de là-bas à la fois(Lamia Missaoui 2003). Les générosités constitutionnelles intégratives de nos États-nations, édifiées au cours de deux ou trois siècles de rapports à l'étranger, à celui qui vient et à qui on offre le choix de « devenir nôtre » ou de repartir, portent de plus en plus à faux: bien des parcours actuels de l'intégration ne sont plus conformes aux modèles historiques ainsi définis.

\section{Des métissages momentanés, partiels}

Robert Ezra Park, préfaçant Everett Stonequist, définissait l'homme marginal en insistant sur le rôle de ces individus, ni d'ici, ni de là-bas, quittant peu à peu leurs appartenances à des collectifs identitaires localement bien spatialisés pour essayer d'en adopter d'autres dans d'autres territoires urbains : ce sont eux qui, éclaireurs et passeurs, anticipaient les cohésions générales entre populations d'origines différentes. Cette conception du 
parcours «de »... « vers »..., qualifié d'intégration, d'insertion, d'acculturation, etc., est très répandue et fait généralement consensus dans les milieux politiques et administratifs, de la ville à la nation. Ce n'est plus ainsi que nous décrivons ces êtres, soumis aux terribles pressions de leurs positions incertaines, intermédiaires entre États différents, présents dans des lieux sans désignation locale. Nous rencontrons plutôt des individus capables d'être d'ici et de là-bas à la fois, contrairement aux descriptions de Park, capables d'entrer-sortir momentanément ou durablement dans des univers de normes qui leur sont étrangers sans pour autant quitter les leurs. L'acte marchand, de haute sociabilité, se décline immédiatement, avec toutes les autres personnes, de la ville ou d'ailleurs, clients locaux ou membres des réseaux. Ainsi des Marocains de la région de Montpellier, arrivés dans les années soixante-dix comme ouvriers agricoles, nous ont dit comment, en quelques jours, ils ont "enfin connu » les «Français », à l'occasion de ces transactions commerciales nouvelles ${ }^{6}$, alors qu'ils avaient vécu des dizaines d'années victimes d'incommensurables distances ségrégatives locales. Nous abordons une sociologie ou une anthropologie des aller-retour, des entrées-sorties, des métissages partiels et momentanés, qui signalent l'apparition de sociabilités autres que celles suggérées par les problématiques des lentes et longues insertions (Lamia Missaoui 1999).

21 Les jeunes filles, peu présentes lors des circulations, réalisent avec intensité ces profils de capacité de sociabilités plurielles: souvent élevées à l'intérieur de la cellule familiale, dans l'intimité féminine, dans une sorte de reproduction des normes des cultures d'origine, elles deviennent très proches des institutions sociales, économiques, etc., des nations d'accueil, celles-là mêmes qui permettent l'insertion, à partir d'accompagnements à la Poste, aux ASSEDIC, de rédactions de demandes et de formulaires divers pour leurs proches analphabètes. Elles sont généralement les comptables, pour la part de «black» comme pour celle d'officialité, des transactions commerciales des parents. Elles effacent avec subtilité les contradictions entre le statut de commerçant transnational des économies souterraines et celui d'ayant-droit de l'aide sociale. Ces dispositions leur ouvrent des perspectives contrastées : pour certaines, une "sortie" rapide des milieux familiaux et une "entrée " non moins rapide dans les sociétés d'accueil, - « sauve qui peut » me disait l'une d'elles - pour d'autres de grandes réussites dans les économies souterraines internationales, non dans la circulation mais dans la gestion des dépôts, commerces, et autres lieux de transaction, d'interface avec les institutions, les commerçants et divers partenaires professionnels locaux.

\section{Des statuts sociaux autres}

Ces diversités et contrastes de trajectoires individuelles concernent aussi les collectifs. Le savoir-être d'ici et de là-bas et l'arrivée massive de "petits migrants » mobilisés par la généralisation de cette forme migratoire nomade, produit des comportements collectifs favorables à la juxtaposition de statuts sociaux contrastés. "Petit ici et notable là-bas » écrivait Lamia Missaoui en 1995 : ces dispositions nous les rencontrons désormais très fréquemment. Les revenus tirés des allers-retours fréquents sont réinvestis dans la région d'origine, et gérés tout aussi familialement ou claniquement que le sont aujourd'hui les dispersions dans l'espace européen. Le projet qui, généralement, justifiait en son temps le départ en migration de tel homme ou de tel couple, est re-élaboré et passe au statut d'une réalité tout autre que celle de la construction au pays d'une maison, jamais achevée, et occupée par d'autres. Des exploitations agricoles sont réaménagées autour d'un outillage qui permet une 
irrigation plus rationnelle et un entretien des sols fréquent, mais aussi par l'usage de semences sélectionnées, en provenance surtout des Pays-Bas et accessoirement diffusées dans les réseaux. De l'outillage pour le bâtiment et les travaux publics, acheté d'occasion en Europe provoque l'apparition d'autant d'entreprises villageoises contribuant à l'aménagement local. Des camions à plate-forme passent souvent les frontières et permettent, dans l'ensemble du Maghreb, de véhiculer de la paille ou du foin de Nord en Sud. Des ateliers artisanaux nombreux se sont ouverts afin de produire par exemple des contre-façons vestimentaires, réimportées lors des remontées. Les micro-investissements productifs se sont généralisés et renforcent le rôle des circulations en les densifiant. Ces mêmes personnes, installées dans diverses nations européennes, qui développent ces initiatives et se «notabilisent » dans leur quartier ou village d'origine, vivent dans les régions d'accueil des statuts précaires, attributaires de revenus minima, ouvriers occasionnels du bâtiment, ou de diverses activités aux statuts aléatoires gérés par des sociétés d'intérim.

\section{Nouvelles hiérarchies sociales}

Ces hiérarchies fracturent verticalement les classiques stratifications sociales et économiques. L'affirmation des valeurs attachées aux réussites dans les commerces, mêlant respect de la parole donnée, notoriété généralisée à l'ensemble des parcours, sens des opportunités, désigne des individus qui bénéficient de possibilités de réussite peu communes : responsabilités morales et commerciales sur tel ou tel produit, dans tel ou tel espace support aux réseaux de circulation, puis installation commerciale de grande ampleur; au fur et à mesure de cette ascension les responsabilités quittent les seuls échanges marchands pour investir des secteurs du religieux dans les villes d'accueil, pour prendre en charge les destins des familiers du village ou du quartier d'origine à l'échelle européenne et au-delà même. Pour ce qui est des Marocains, et aussi des Africains subsahariens, ces nouvelles possibilités sont en train de transformer le sens même des cheminements classiques de l'intégration définis et offerts par les États-nations européens. Les contrastes constitutionnels entre les divers modèles nationaux, communautaires, «jacobins», ethniques, qui caractérisent l'Europe, les singularités différenciatrices des diverses histoires ne s'imposent plus comme des préalables incontournables au maintien résidentiel de ces populations ni à leur circulation. L'invention des «beurs» en France dans les années quatre-vingt, définis comme orphelins de pères inassimilables, et donc relevant d'un grand dessein d'intégration, apparait aujourd'hui à ces populations comme un subterfuge développé par des autorités désemparées de ne pouvoir façonner les destins de ces jeunes : leurs pères, soi-disant absents, créaient alors toutes les conditions nécessaires au déploiement actuel. Ils provoquaient le basculement du modèle d'intégration des diasporas, fidèles à leurs origines certes, mais rapidement assimilées par la vie économique - sur le mode de la complémentarité (Alain Médam, 1993 - sociale et politique, vers un nouveau modèle de collectifs migratoires nomades - attachés exclusivement, jusque dans leurs activités de migrants commerciaux, à leurs milieux et à leurs lieux d'origine, mais néanmoins capables de développer en de multiples étapes leur présence dans l'internationalité européenne. Les migrants qui demeurent soumis aux formes classiques de l'exploitation de la force internationale de travail et à l'enfermement dans les étroites limites des entreprises qui les emploient et des villes qui les hébergent, toujours très nombreux, numériquement très dominants, bien sûr, échappent de plus en plus eux-aussi aux intentions intégratives : ils se savent en situation d'infériorité par rapport à leur passé dans les activités de réseaux et, avec ou 
sans papiers, ont de plus en plus tendance à se considérer comme en situation d'errance, perdant de vue l'ici et le là-bas, au bénéfice d'une sorte de relocalisation dans un espace religieux. Cette tendance se généralise d'autant plus que les uns et les autres résident en commun.

L'institution des pouvoirs sur ces territoires des circulations est fort différente de celle des États-nations, des sociétés de sédentarité. Les "notaires informels", témoins et gardiens des paroles échangées lors des cooptations, disposent d'un pouvoir bien réel, mais tributaire d'équilibres délicats: disons succinctement que l'étendue de leur influence dépend de leur capacité d'entretenir des relations avec les pouvoirs locaux, politiques et policiers, avec les représentants officiels des États d'origine des populations de migrants, avec leurs représentants religieux, avec des milieux commerciaux de l'officialité et de la subterranéité, avec encore les milieux troubles des divers trafics criminels. Ils disposent en quelque sorte d'un statut qui les situe au-delà des exigences de l'honneur engagé dans les échanges d'oralité, par le fait même qu'ils ont souvent à protéger l'éthique des réseaux de migrants commerçants de celle - si l'on peut dire - des réseaux mafieux et/ou criminels ; ils sont à cheval sur de nombreuses frontières de normes et d'intérêts. À la fois craints par leurs divers partenaires, leur alliance est recherchée par tous : si, à une extrémité de l'organisation sociale et politique, les sphères de l'officialité espèrent ainsi maîtriser le caractère profondément subversif de ces formes sociales peu saisissables et sans institutions territorialisées - inadmissibles contre-modèles - les réseaux mafieux, eux, en attendent une aide, envieux qu'ils sont de leurs capacités de circulation. Mais il s'agit bien d'équilibres entre eau et feu: l'institutionnalisation étatique et le cloisonnement extrême des réseaux mafieux sont essentiellement différents, autres. Les "notaires informels", ces hommes investis de pouvoirs uniques dans les cooptations des circulants et les régulations de leurs activités comme de leurs mobilités, disparaissent dès lors qu'ils ne maintiennent plus l'équilibre entre attaches diverses, qu'ils s'associent trop ouvertement à l'un ou l'autre de leurs partenaires. Jusqu'en 1997, alors que l'Italie et l'Espagne n'hébergeaient pas de centralité des réseaux, mais des circulations, des passages, et supportaient essentiellement une migration récente de main-d'œuvre ouvrière, les cas d'exclusion de "notaires informels » que nous avons eus à connaittre concernaient des Algériens à Belsunce en 1989-1990, trop attachés au F.L.N. et à l'Amicale des Algériens en France : Le Front Islamique du Salut, relayé par des trabendistes, tentait de placer ses propres hommes dans ces positions de pouvoir. Pendant quelques mois des personnes liges tentèrent de maintenir les équilibres des réseaux. Peine perdue, les transactions et les partenariats se multipliaient, pour le plus grand développement des réseaux en cours de mondialisation, Polonais, Bulgares, Turcs, Italiens. Le repli islamiste que supposait ce profil nouveau des « notaires informels » fut refusé : il contrecarrait la nécessaire ouverture des uns et des autres vers une éthique de la parole intermédiaire aux diverses croyances, aux nombreuses origines et donc il gênait le développement, l'expansion des réseaux vers l'internationalité. La mosquée qui avait supplanté les bistrots de la Canebière comme nouveau lieu de rencontres avec les «notaires informels » garda sa fonction, mais ceux qui s'imposèrent majoritairement (3 sur 5 identifiés par nos enquêtes à Marseille) furent désormais et jusqu'en 1995 environ, des hadjs Sénégalais, beaucoup plus ouverts aux altérités de leurs partenaires, et non moins proches des pouvoirs policiers locaux (Daouda Koné, 1996). Ils représentaient de plus une manifestation nouvelle et en plein essor des réseaux de circulants, de diverses origines africaines subsahariennes, vers la France et l'Angleterre, via le Maroc et l'Espagne. La proximité habituelle avec les réseaux policiers les condamna: ils s'impliquèrent dans les trafics d'héroïne et de cocaïne de provenance africaine et ne purent échapper au regard 
et à l'action de leurs « partenaires » policiers. Des arrestations s'en suivirent et, surtout, pourraiton dire, du point de vue des réseaux, une perte de confiance radicale dans leur rôle et une fracture entre réseaux africains et réseaux à dominante marocaine. Ces troubles parmi les " notaires informels ", même s'ils durèrent peu, suffirent pour introduire en Espagne et en Italie, qui commençaient à héberger des réseaux de migrants commerçants, de grandes confusions dans la différenciation entre réseaux de migrants commerçants et réseaux mafieux. Les Marocains qui, depuis le début des années quatre-vingt-dix, développaient une migration puissante, peu concernés dans leurs transactions par les réseaux africains, isolèrent, dans leurs nouvelles installations en Espagne, ceux qu'ils appelèrent désormais en arabe «les bandes noires", pour le plus grand malheur des migrants africains subsahariens sans papiers condamnés à un supplément de solitude. De nouveaux «notaires informels» apparurent, de Milan à Marrakech, tous entrepreneurs de l'officialité ayant réussi dans des activités préalables de migrants commerçants, mais dans une diversité de gestion extrêmement contrastée des espaces sous leur « juridiction morale », reflétant, comme jamais auparavant, la réalité des compositions, des négociations, entre territoires circulatoires et territoires locaux. Ainsi se diversifient actuellement, le long des territoires circulatoires, des « régions morales » distinctes des régions politiques européennes auxquelles elles se superposèrent. De telle sorte que, depuis les accords de Schengen, apparaissent, des « frontières » originales différenciant des pratiques des réseaux en même temps qu'identifiant une «carte » des régions migratoires de l'Europe sans coïncidence avec les totalités nationales et leurs frontières historiques. La riche mais obscure notion de «district moral $»^{7}$ proposée par Robert Ezra Park afin d'expliquer comment dispositions individuelles et histoire de la ville produisaient les transformations urbaines les plus déterminantes, à l'aide de diverses mobilités locales, par la subversion morale et éphémère de lieux précis, cette notion donc, à condition d'un élargissement aux mobilités internationales, trouve un usage opportun à la compréhension de ces phénomènes de contournement des « logiques Schengen » dès leur apparition.

\section{La gestion des confins}

D’Algésiras à Marseille la frontière entre zonages des réseaux est à Alicante : là se rejoignent les Algériens venant par Oran, les Marocains et les populations subsahariennes passant par le détroit de Gibraltar. Alicante, avec Valencia, est un débouché historique de Madrid vers la Méditerranée. Cette ville, porte méridionale de la Catalogne, développe une rare capacité de gestion pacifique des cosmopolitismes, l'aventure de l'aller-retour des Pieds-Noirs en Algérie (Juan-David Sempere, 1999), les représentations festives valorisantes des « Maures » lors des fêtes semestrielles des Moros $i$ Cristianos alors même que vers Alméria, $200 \mathrm{kms}$ plus au sud, en Andalousie, les populations locales commettent des exactions xénophobes contre les migrants marocains, cette ville accueille environ trente «bazars», commerces de produits divers, tenus par des Marocains et des Algériens; ces boutiques, proches du port, sont associées à une quarantaine d'autres, dans la petite ville de Crevillent, à trente kilomètres à l'ouest le long de l'autoroute menant de Marseille à Algésiras. Cette centralité nouvelle, depuis 1997, des réseaux commerciaux, s'institue "frontière des commerces propres », selon les dires de deux «notaires informels » rencontrés longuement à Murcia et opérant sur cette «centralité-frontière ». Au Sud, nous avons rencontré trois de ces notaires, institués tels lors de la période de gestion "africaine » des réseaux: deux d'entre eux, à Grenade et à Malaga, ont développé, en accord évident avec les polices locales et les polices marocaines, avec les autorités musulmanes, les trafics de jeunes femmes marocaines pour la prostitution ${ }^{8}$ sur la Costa del Sol et les grandes villes du Sud 
espagnol, les trocs entre marchandises revendues sur les marchés publics - chaussures de sport, vêtements - et haschich « remonté » du Rif marocain par quintaux, le contrôle des Hollandais livrant la cocaïne aux prostituées et aux boîtes de nuit, mais aussi la vente en bordure d'autoroute de produits de consommation courante complétant en dernière heure les chargements des migrants commerçants. Ils fixent également, afin de disposer de viviers pour leurs trafics, des sortes de réserves de migrants en situation d'errance, recrutés parmi les sanspapiers, et cédés à la journée, pour des salaires dérisoires, aux propriétaires agricoles des serres de cultures sur-intensives, ajoutant ainsi à leur misère l'exploitation féroce de leurs solitudes. Dans les premiers mois de leur migration en Espagne ils ont collaboré avec des Africains subsahariens, Nigérians, Gambiens et Sénégalais, dans l'acheminement du Maroc vers l'Europe, d'héroïne, qui subissait une dernière transformation dans la région de Grenade. Ces « notaires informels » sont des interlocuteurs très valorisés par les autorités politiques et policières régionales et locales et participent activement à la vie des mosquées naissantes dans les grandes villes du Sud. Ils contribuent à l'institutionnalisation, à l'intérieur de l'espace Schengen, de zones troubles, de confins, comme en identifient des chercheurs italiens dans la région de Trieste ou de Barri, en Sicile, à Naples et dans des faubourgs milanais. Les tensions entre la Communauté européenne et le Maroc à propos des passages d'Africains subsahariens, et de l'apparition à Rabat, Casablanca et Tanger, des premiers villages urbains africains, ne sont qu'une expression prudente, aujourd'hui possible, des problèmes beaucoup plus vastes et criminels du Sud de l'Espagne : une pseudo- tentative de résolution de cette situation se traduirat-elle par une injonction politique de forme coloniale, que lesaccusations actuellement portées contre le royaume chérifien laissent augurer? L'Espagne et l'Europe semblent vouloir en effet exporter ces troubles dans les pays extérieurs riverains, et particulièrement vers le Maroc. Ainsi l'idéologie sécuritaire présentée comme nécessaire aux régulations de l'espace Schengen contribuerait-elle à la désagrégation des pays riverains pauvres, en transformant leur situation de " portes migratoires » en réservoirs de misère mondialisés.

D'Alicante à Marseille fonctionnent toujours les réseaux sous la forme paisible précédemment décrite; des centralités locales scandent ces espaces en autant d'étapes avec leurs désormais classiques concentrations résidentielles communautaires, et leurs commerces locaux, à Valencia, Tarragone, Barcelone, Perpignan, Montpellier, Nîmes, etc. La séparation avec les réseaux de trafic de produits d'usage illicite y est nettement pratiquée. Les réseaux mafieux se sont reconstitués autour de la modification de la frontière franco-espagnole, transformée de frontière-barrière en un vaste espace sous contrôle douanier, de Gérone à Montpellier. Les Étatsnations européens, dans leur tentative de fédération, ne savent pas davantage gérer leurs transformations frontalières que leurs confins.

Révélateurs et acteurs de ces transformations de fait, les réseaux de migrants entrepreneurs commerciaux participent de l'accélération contemporaine des circulations mondiales. Saisissant les opportunités de libéralisation des circulations, nécessaires à la mondialisation opérée par les acteurs de l'officialité à partir de leurs compétences techniques, les migrants que nous évoquons dans cet article affirment d'abord une compétence sociale, relationnelle, signalant une nature antagonique de leurs réseaux avec ceux des économies de l'officialité .

En ce sens dès aujourd'hui, et probablement en préalable à de profondes transformations des ordres institués, ils sont aussi dérangeants pour le monde qu'ils le sont pour l'Étatnation. 


\section{NOTES}

1. En association, souvent, avec Juan David Sempere, chercheur à l'université d'Alicante.

2. Les " notaires informels", personnes référentes lors des transactions commerciales, veillent à la régularité des échanges, au respect de la parole donnée, à la bonne répartition des flux de clients : leur dénonciation d'entorses aux règles de bonne conduite produisent une éviction immédiate de commerçants et de «fourmis ».

3. Pour ce que nous avons pu en savoir, il s'agit de passages de personnes (écrivains engagés et prédicateurs) prenant momentanément leur distance du territoire algérien.

4. Alain Tarrius Les fourmis d'Europe. Migrants pauvres, migrants riches et nouvelles villes internationales, Paris,L'Harmattan, 1992.

5. On reconnaîtra dans les huit lignes qui suivent quelques positions clefs de Maurice Halbwachs, notamment en ce qui concerne le rapport entre lieux, identités et mémoire collective; en particulier exprimées dans La topographie légendaire des Évangiles en terre Sainte, (Paris, PUF, 1942.). Toutefois nous divergeons sur deux points essentiels : la référence aux lieux, première pour Halbwachs, doit selon notre point de vue laisser davantage place aux temporalités sociales, et le fonctionnement de la mémoire collective relève moins de l'entassement, du puits mnésique, que de l'incessante reformulation. C'est ainsi qu'Halbwachs ne sait pas situer l'amnésie. Nous trouvons sur ces point plus opportunes les positions du phénoménologue G. Husserl, Crise des sciences sociales et phénoménologie transcendantale, Paris,Gallimard, (traduction) 1973.

6. Alors que les passages aux frontières entre l'Espagne et le Maroc doublaient, de un million à deux millions environ entre 1991 et 2000, (Rabat, Fondation Hassan II et Centre Jacques Berque, juillet 2001) plus de $60 \%$ des migrants marocains arrivés dans la Région Languedoc-Roussillon avant 1985, essentiellement pour le travail agricole, se re-localisaient dans les grandes villes et passaient aux économies transfrontalières (Raymond Sala et Alain Tarrius Migrants d'hier et d'aujourd'hui en Roussillon, Canet, Ed. Trabucaire, 2000).

7. Nous reprenons la traduction proposée par Isaac Joseph et acceptée par la communauté des anthropologues et sociologues de la ville, bien que nous préférions celle de "zones de mœurs ».

8. Fatima Lahbabi : «La migration des femmes marocaines en Andalousie », Thèse de doctorat de sociologie, Université de Toulouse-le-Mirail. 2003.

\section{AUTEUR}

\section{ALAIN TARRIUS}

Université Toulouse Le Mirail 